\title{
Comparison of RANS and LES in the Prediction of Airflow Field over Steep Complex Terrain
}

\author{
Takanori Uchida ${ }^{*}$, Graham $\mathbf{L i}^{2}$ \\ ${ }^{1}$ Research Institute for Applied Mechanics (RIAM), Kyushu University, Fukuoka, Japan \\ ${ }^{2}$ Tsubasa Windfarm Design, Tokyo, Japan \\ Email: ^takanori@riam.kyushu-u.ac.jp
}

How to cite this paper: Uchida, T. and $\mathrm{Li}$, G. (2018) Comparison of RANS and LES in the Prediction of Airflow Field over Steep Complex Terrain. Open Journal of Fluid Dynamics, 8, 286-307.

https://doi.org/10.4236/ojfd.2018.83018

Received: November 13, 2017

Accepted: August 31, 2018

Published: September 3, 2018

Copyright (c) 2018 by authors and Scientific Research Publishing Inc. This work is licensed under the Creative Commons Attribution International License (CC BY 4.0).

http://creativecommons.org/licenses/by/4.0/

(c) (i) Open Access

\begin{abstract}
The present study compared the prediction accuracy of the three CFD software packages for simulating airflow around a three-dimensional, isolated hill with a steep slope: 1 ) WindSim (turbulence model: RNG $k$ - $\varepsilon$ RANS), 2) Meteodyn WT (turbulence model: $k-L$ RANS), which are the leading commercially available CFD software packages in the wind power industry and 3) RIAM-COMPACT (turbulence model: standard Smagorinsky LES), which has been developed by the lead author of the present paper. Distinct differences in the airflow patterns were identified in the vicinity of the isolated hill (especially downstream of the hill) between the RANS results and the LES results. No reverse flow region (vortex region) characterized by negative wind velocities was identified downstream of the isolated hill in the result from the simulation with WindSim (RNG $k-\varepsilon$ RANS) and Meteodyn WT ( $k-L$ RANS). In the case of the simulation with RIAM-COMPACT natural terrain version (standard Smagorinsky LES), a reverse flow region (vortex region) characterized by negative wind velocities clearly forms. Next, an example of wind risk (terrain-induced turbulence) diagnostics was presented for a large-scale wind farm in China. The vertical profiles of the streamwise $(x)$ wind velocity do not follow the so-called power law wind profile; a large velocity deficit can be seen between the hub center and the lower end of the swept area in the case of the LES calculation (RIAM-COMPACT).
\end{abstract}

\section{Keywords}

RANS, LES, Isolated Hill, Large-Scale Wind Farm

\section{Introduction}

We have developed an unsteady and non-linear wind synopsis simulator called RIAM-COMPACT (Research Institute for Applied Mechanics, Kyushu Univer- 
sity, Computational Prediction of Airflow over Complex Terrain) in order to simulate the airflow on a microscale, i.e., a few tens of $\mathrm{km}$ or less [1]-[13]. In RIAM-COMPACT, a large-eddy simulation (LES) has been adopted for turbulence modeling. LES is a technique in which the structures of relatively large eddies are directly simulated and smaller eddies are modeled using a sub-grid scale model. Efforts have been made to promote RIAM-COMPACT, mainly in the wind power industry (e.g., private wind power providers, local governments, and wind turbine manufacturers) in Japan. Computation time had been an issue of concern for the RIAM-COMPACT software, which focuses on unsteady turbulence simulations (LES). The present fluid simulation solver is compatible with multi-core CPUs such as the Intel Core i9 and also with GPGPU, which has drastically reduced the computation time, leaving no appreciable problems in terms of the practical use of the RIAM-COMPACT software.

On another front, commercially available CFD software such as STAR-CCM+ [14] and ANSYS (CFD, Fluent, CFX) [15] has developed mainly as an engineering tool primarily in the automobile and aviation industries until the present time. Recently, some of the above-mentioned general purpose thermal fluid analysis software has started being adopted in the wind power industry. In the previous study [16] [17] [18], the simulation results obtained from the RIAM-COMPACT software were compared to those from STAR-CCM+, one of the leading commercially available CFD software packages. The results of the comparison are discussed. In addition, open-source CFD software packages are more widely used than in the past. One of the most widely used software packages is OpenFOAM (OpenField Operation And Manipulation) [19]. OpenFOAM is an open-source CFD toolbox which has been released and distributed under the GNU GPL (General Public License) [20] by the OpenFOAM Foundation, a non-profit organization. In the previous study [21], the simulation results obtained from the RIAM-COMPACT software were also compared to those from OpenFOAM, and the results of the comparison are discussed.

The wind power industry has on its own developed and distributed CFD software designed for selecting sites appropriate for the installation of wind turbine generators. One such leading software package is Meteodyn WT [22], which has been developed by Meteodyn in France. Meteodyn WT is a CFD software package which incorporates a RANS turbulence equation with a one-equation closure scheme ( $k-L$ turbulence model; here, $k$ and $L$ refer to turbulence energy and the turbulence length scale, respectively). On October 12, 2017, "WT6.0", the latest version of the software, was released. Another one of the most widely used software packages is WindSim [23] by Norway-based WindSim AS. WindSim is a CFD software package which uses a RANS turbulence model and has been designed specifically for wind resource assessment. In December 2016, the latest version of the company's software package, WindSim 8.0, was released. These two CFD software packages are specialized for wind power resource assessment as well as the RIAM-COMPACT CFD software package. 
In the present study, numerical simulations are performed for airflow over and around a three-dimensional, isolated hill with a steep slope angle using the three CFD software packages (WindSim, Meteodyn WT and RIAM-COMPACT). The results of the comparison are discussed. Next, the numerical simulations for airflow over a large-scale wind farm in China [21] are performed with RIAM-COMPACT, which is based on an LES turbulence model, and Meteodyn WT, which is based on a RANS turbulence model.

\section{Overview of the Software Packages (RIAM-COMPACT and WindSim) and Numerical Simulation Set-Up in the Case of a Three-Dimensional, Isolated Hill with a Steep Slope Angle}

The numerical wind simulations in the present study are conducted for high Reynolds number airflow over and around a three-dimensional, isolated hill with a steep slope angle and a large-scale wind farm in China. Table 1 shows the simulation set-ups adopted in the two software packages which are used in

Table 1. Comparison of numerical simulation methods, parameters, and settings between the two software packages.

\begin{tabular}{|c|c|c|}
\hline CFD model & RIAM-COMPACT & WindSim \\
\hline Turbulence model & Standard Smagorinsky LES & RNG $k$ - $\varepsilon$ RANS \\
\hline $\begin{array}{l}\text { Atmospheric stratification } \\
\text { (Atmospheric stability) }\end{array}$ & \multicolumn{2}{|c|}{ Neutral atmosphere } \\
\hline Coriolis force & \multicolumn{2}{|c|}{ Not considered } \\
\hline Surface roughness & $\begin{array}{l}\text { Not considered } \\
\text { (Smooth surface) }\end{array}$ & Roughness length: 0.001 \\
\hline $\begin{array}{l}\text { Ground surface } \\
\text { boundary condition }\end{array}$ & $\begin{array}{l}\text { Non-slip condition } \\
\text { (Three wind velocity components } \\
\text { at the ground surface are zero.) }\end{array}$ & Wall function \\
\hline $\begin{array}{l}\text { Shape function of } \\
\text { the isolated hill } z(r)\end{array}$ & \multicolumn{2}{|c|}{$0.5 h \times\{1+\cos (\pi r / a)\}, r=\left(x^{2}+y^{2}\right)^{1 / 2}, a=2 h$} \\
\hline Height of the isolated hill $h$ & \multicolumn{2}{|l|}{$100(\mathrm{~m})$} \\
\hline Reynolds number $\operatorname{Re}\left(=U_{\text {in }} h / v\right)$ & $5 \times 10^{4}$ and $1 \times 10^{7}$ & $5 \times 10^{4}$ and $1 \times 10^{7}$ \\
\hline Time step $\Delta t$ & $\begin{array}{l}10^{-3} h / U_{\text {in }}(\mathrm{s}) \text { for } \mathrm{Re}=5 \times 10^{4} \\
10^{-7} h / U_{\text {in }}(\mathrm{s}) \text { for } \mathrm{Re}=1 \times 10^{7}\end{array}$ & - \\
\hline Computational domain size & $\begin{array}{c}13 h(i) \times 9 h(j) \times 8 h(k) \\
\text { for } \operatorname{Re}=5 \times 10^{4} \\
19 h(i) \times 18 h(j) \times 8 h(k) \\
\text { for } \operatorname{Re}=1 \times 10^{7} \\
325(i) \times 226(j) \times 37(k) \\
\text { (Approx. } 2.7 \text { million points) }\end{array}$ & $13 h(i) \times 9 h(j) \times 8 h(k)$ \\
\hline $\begin{array}{c}\text { Number of } \\
\text { computational grid points }\end{array}$ & $\begin{array}{c}\text { for } \operatorname{Re}=5 \times 10^{4} \\
436(i) \times 325(j) \times 101(k) \\
\text { (Approx. } 14.3 \text { million points) } \\
\text { for } \operatorname{Re}=1 \times 10^{7}\end{array}$ & $\begin{array}{c}325(i) \times 226(j) \times 37(k) \\
\text { (Approx. } 2.7 \text { million points) }\end{array}$ \\
\hline $\begin{array}{c}\text { Streamwise }(x) \text { grid spacing }(\Delta x) \\
\text { Spanwise }(y) \text { grid spacing }(\Delta y)\end{array}$ & $\begin{array}{c}0.04 \times h \text { for } \operatorname{Re}=5 \times 10^{4} \\
(0.035-0.5) \times h \text { for } \operatorname{Re}=1 \times 10^{7}\end{array}$ & $0.04 \times h$ \\
\hline Vertical $(z)$ grid spacing $(\Delta z)$ & $\begin{array}{c}(0.05-0.40) \times h \text { for } \operatorname{Re}=5 \times 10^{4} \\
(0.000004-0.6) \times h \text { for } \operatorname{Re}=1 \times 10^{7}\end{array}$ & $(0.05-0.40) \times h$ \\
\hline
\end{tabular}


the present study: RIAM-COMPACT natural terrain version (turbulence model: LES) and WindSim (turbulence model: RANS). Figure 1 and Figure 2 illustrate the full computational grid used for WindSim and an enlarged view of the grid in the vicinity of the isolated hill, respectively. Figure 3 shows the inflow profile used for all of the simulations in the present study. Figure 4 shows the characteristic wind velocity and length scales which are employed for the simulations with RIAM-COMPACT.

In RIAM-COMPACT, a collocated grid in a general curvilinear coordinate system is used in order to numerically predict local wind flow over complex terrain with high accuracy while avoiding numerical instability. For the numerical simulation method, a FDM is adopted, and an LES model is used for the turbulence model. For the computational algorithm, a method similar to a FS method [24] is used, and a time marching method based on the Euler explicit method is adopted. The Poisson's equation for pressure is solved by the SOR method.

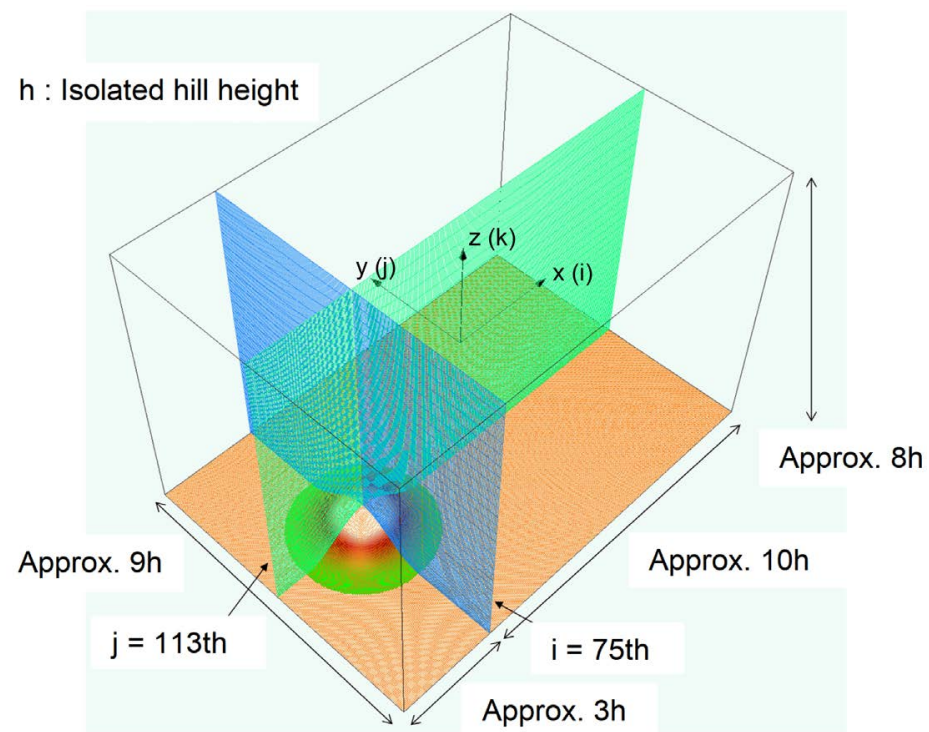

Figure 1. Computational grid used in the simulations with WindSim, $\operatorname{Re}=5 \times 10^{4}$ and 5 $\times 10^{7}$.

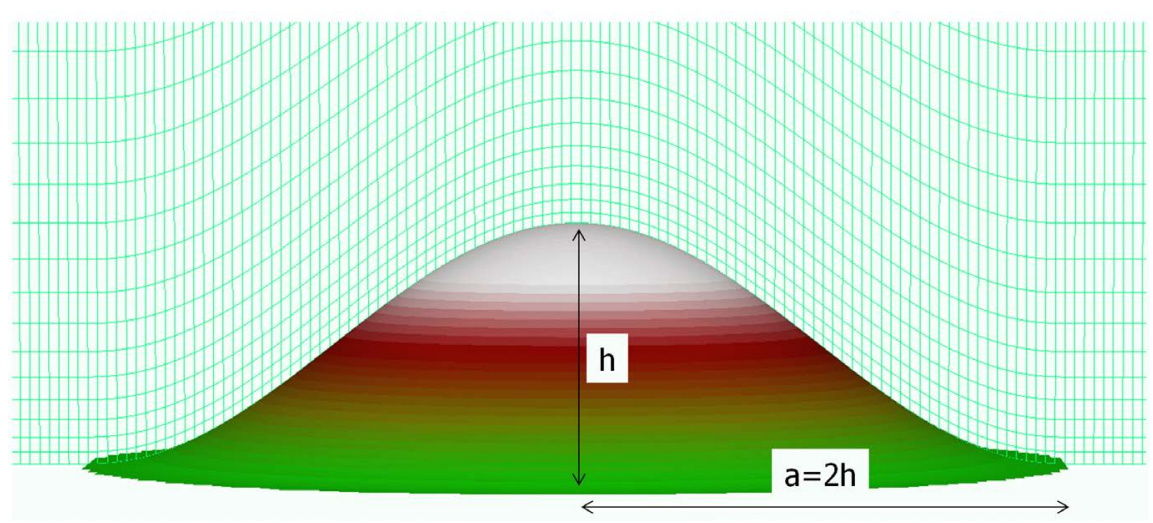

Figure 2. Enlarged view of the computational grid used in the simulations with WindSim, $\operatorname{Re}=5 \times 10^{4}$ and $5 \times 10^{7}$. 


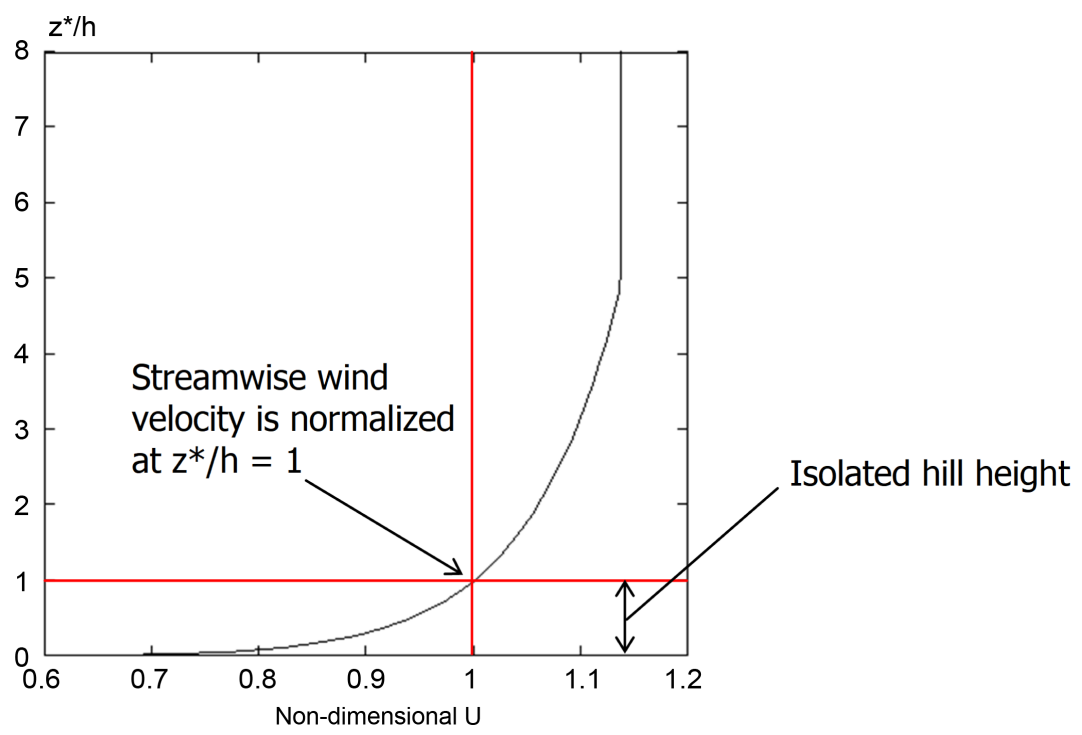

Figure 3. Inflow wind velocity profile used in the present study.

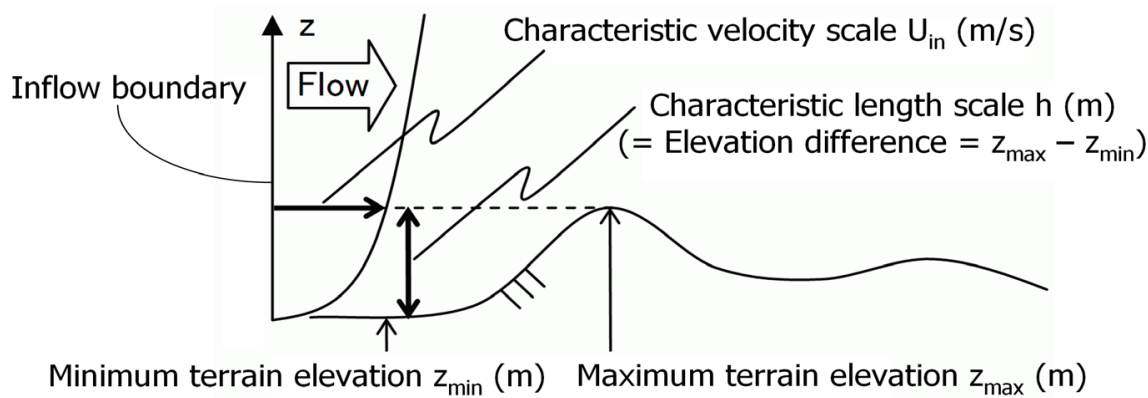

Figure 4. Characteristic wind velocity and length scales used in the simulation with RIAM-COMPACT.

For discretization of all the spatial terms in the governing equations except for the convective term in the Navier-Stokes equation, a second-order central difference scheme is applied. For the convective term, a third-order upwind difference scheme is used. An interpolation technique based on four-point differencing and four-point interpolation by Kajishima [25] is used for the fourth-order central differencing that appears in the discretized form of the convective term. For the weighting of the numerical diffusion term in the convective term discretized by third-order upwind differencing, $\alpha=3.0$ is commonly applied in the Kawamura-Kuwahara scheme [26]. However, $\alpha=0.5$ is used in the present study to minimize the influence of numerical diffusion. For the LES subgrid-scale modeling, the standard Smagorinsky model [27] is adopted with a model coefficient of 0.1 in conjunction with a wall-damping function. For further details of the numerical simulation techniques, refer to Uchida [1]-[13].

Regarding the boundary conditions adopted for the simulations with RIAM-COMPACT, the same inflow profile as used for the simulations with WindSim (Figure 3 ) is given at the inflow boundary. At the side and upper boundaries, free-slip conditions are applied, and convective outflow conditions 
are applied at the outflow boundary. On the ground surface, a non-slip boundary condition is imposed. For the simulation at $\operatorname{Re}\left(=U_{\text {in }} h / v\right)=10^{7}$, the number of grid points is changed to 101 in the vertical direction, and the minimum vertical grid spacing in is set to $\Delta z_{\min } / h=4 \times 10^{-7}$ according to the equation below (see Table 1):

$$
\Delta z_{\min } / h=\frac{0.1}{\sqrt{\mathrm{Re}}}
$$

In contrast to RIAM-COMPACT, WindSim uses RANS models. In the present study, the RNG $k-\varepsilon$ RANS model is selected for the simulations. Refer to [23] for the numerical simulation methods used in WindSim and other details about this software.

\section{Comparison of the Simulation Results from the Two CFD Software Packages (RIAM-COMPACT and WindSim) in the Case of a Three-Dimensional, Isolated Hill with a Steep Slope Angle}

Figure 5 shows the ensemble-averaged flow fields from the simulations with WindSim (turbulence model: RNG $k-\mathcal{E}$ RANS). In neither of these simulations

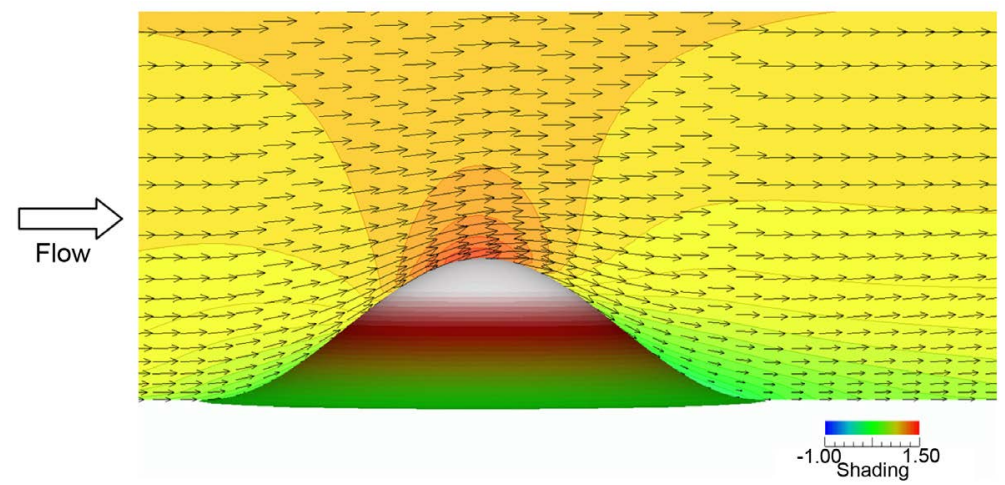

(a)

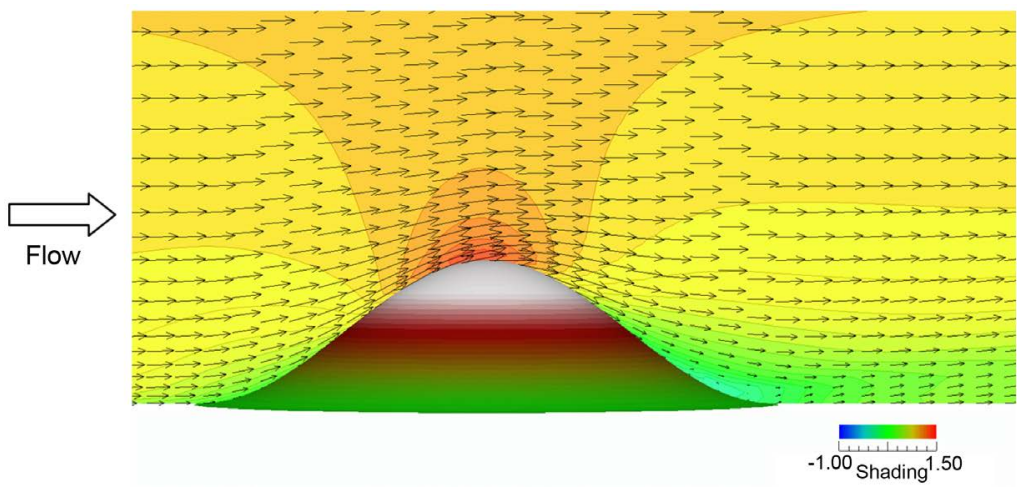

(b)

Figure 5. Wind velocity vectors and contour of streamwise $(x)$ wind velocity (non-dimensional) on the $\mathrm{x}-\mathrm{z}$ cross-section at the center of the span $(\mathrm{y}=0)$, ensem-

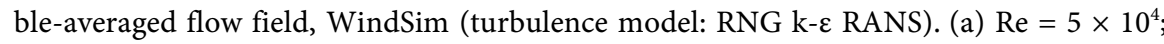
(b) $\operatorname{Re}=5 \times 10^{7}$. 
$\left(\operatorname{Re}=5 \times 10^{4}\right.$ and $\left.5 \times 10^{7}\right)$ did a reverse flow region (vortex region), in which the values of the streamwise wind velocity are negative, form downstream of the isolated hill. Instead, a potential-flow-like pattern formed in both simulations.

Figure 6 shows instantaneous flow fields from the simulations with RIAM-COMPACT (turbulence model: standard Smagorinsky LES) ( $\mathrm{Re}=5 \times 10^{4}$ and $\left.1 \times 10^{7}\right)$. An examination of these simulation results reveals the clear presence of a reverse flow region (vortex region), in which the values of the streamwise wind velocity are negative, downstream of the isolated hill.

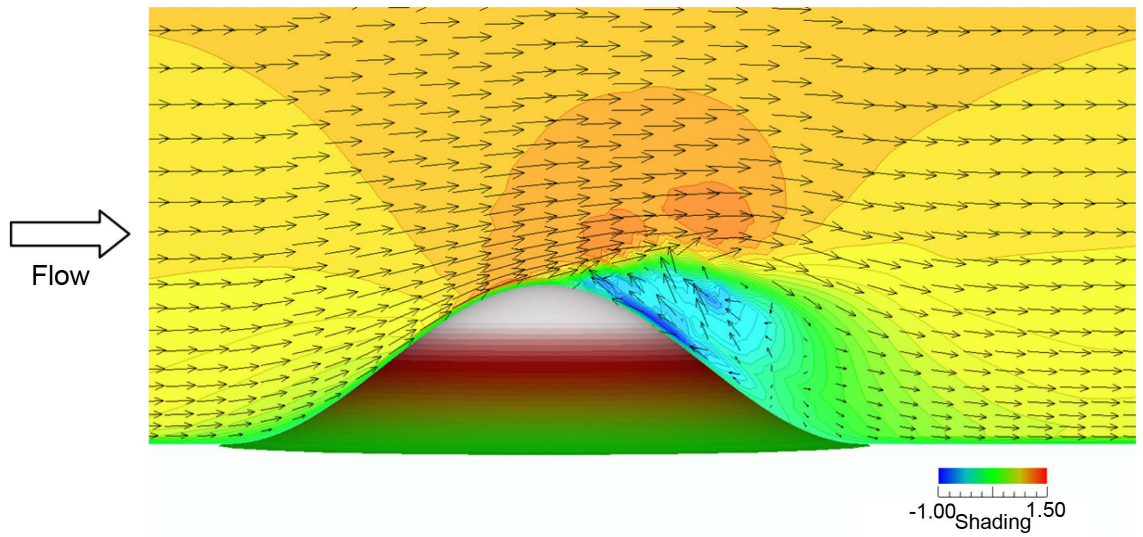

(a)

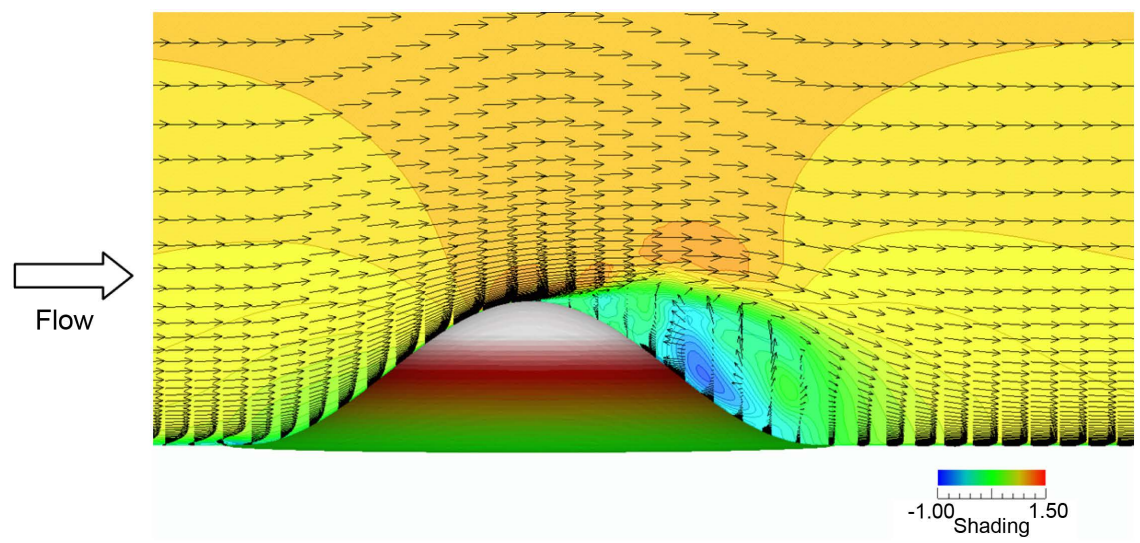

(b)

Figure 6. Wind velocity vectors and contour of streamwise (x) wind velocity (non-dimensional) on the $\mathrm{x}-\mathrm{z}$ cross-section at the center of the span $(\mathrm{y}=0)$, instantaneous flow field, RIAM-COMPACT (turbulence model: standard Smagorinsky LES). (a) Re $=5 \times 10^{4}$, non-dimensional time $=5.0 ;(b) \mathrm{Re}=1 \times 10^{7}$, non-dimensional time $=7.0$.

\section{Overview of the Software Packages (RIAM-COMPACT and Meteodyn WT) and Numerical Simulation Set-Up in the Case of a Three-Dimensional, Isolated Hill with a Steep Slope Angle}

For the present study, numerical simulations are conducted for high Reynolds number flow around a three-dimensional, isolated hill with a steep slope angle using RIAM-COMPACT, which is based on an LES turbulence model, and 
Meteodyn WT, which is based on a RANS turbulence model (see Table 2). Figure 7 shows the computational domain and grid used for the simulation with Meteodyn WT. An enlarged view of the computational grid in the vicinity of the isolated hill from the simulation with Meteodyn WT is shown in Figure 8. Figure 9 shows the method used to set the inflow profile in Meteodyn WT and the inflow profile generated for the present study.

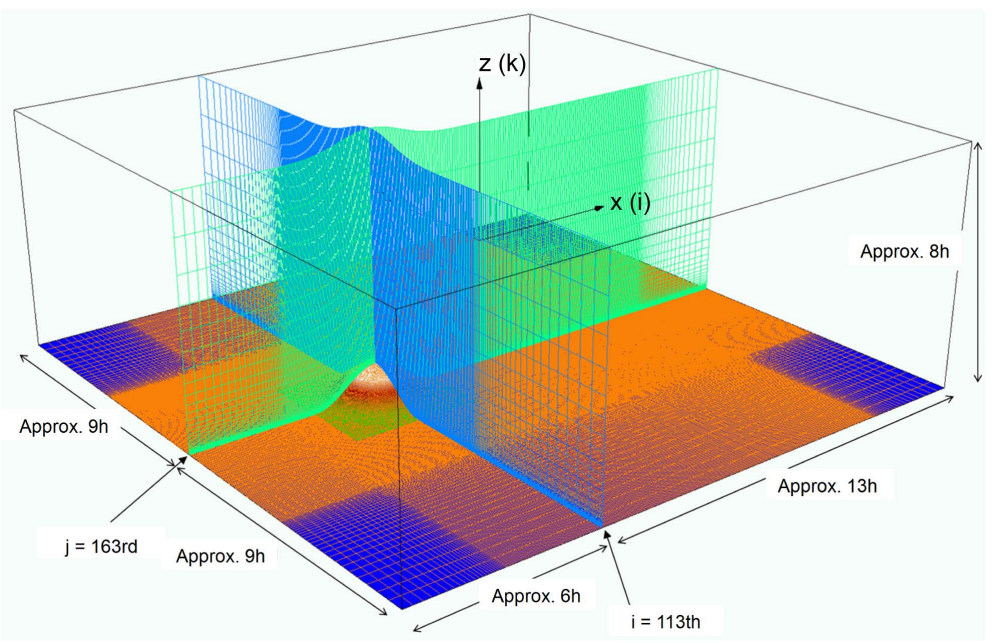

Figure 7. Computational domain and grid used for the simulation with Meteodyn WT, $\operatorname{Re}=10^{7}$.

Table 2. Comparison of numerical simulation methods, parameters, and settings between the two software packages.

\begin{tabular}{|c|c|c|}
\hline CFD model & RIAM-COMPACT & Meteodyn WT \\
\hline Turbulence model & $\begin{array}{c}\text { Standard } \\
\text { Smagorinsky LES }\end{array}$ & $\begin{array}{c}k \text { - } L \text { RANS } \\
\text { (A single equation model) }\end{array}$ \\
\hline $\begin{array}{l}\text { Atmospheric stratification } \\
\text { (Atmospheric stability) }\end{array}$ & \multicolumn{2}{|c|}{ Neutral atmosphere } \\
\hline Coriolis force & \multicolumn{2}{|c|}{ Not considered } \\
\hline Surface roughness & $\begin{array}{l}\text { Not considered } \\
\text { (Smooth surface) }\end{array}$ & $\begin{array}{l}\text { Roughness length: } 0.05 \\
\text { (For the ground surface not } \\
\text { on the isolated hill: } 0.001 \text { ) }\end{array}$ \\
\hline $\begin{array}{l}\text { Ground surface } \\
\text { boundary condition }\end{array}$ & $\begin{array}{l}\text { Non-slip } \\
\text { (Three wind velo } \\
\text { at the ground su }\end{array}$ & $\begin{array}{l}\text { ondition } \\
\text { ity components } \\
\text { rface are zero.) }\end{array}$ \\
\hline Shape function of the isolated hill $Z(r)$ & $0.5 h \times\{1+\cos (\pi r / a)\}$ & $=\left(x^{2}+y^{2}\right) 1 / 2, a=2 h$ \\
\hline Height of the isolated hill $h$ & \multicolumn{2}{|c|}{$100(\mathrm{~m})$} \\
\hline Reynolds number $\operatorname{Re}\left(=U_{\text {in }} h / v\right)$ & $10^{6}$ & $10^{7}$ \\
\hline Time step $\Delta t$ & $10^{-5} h / U_{\text {in }}(\mathrm{s})$ & - \\
\hline Computational domain size & \multicolumn{2}{|c|}{$19 h(i) \times 18 h(j) \times 8 h(k)$} \\
\hline Number of computational grid points & $\begin{array}{c}436(\hat{I}) \times 325(j) \times 101(k) \\
\text { (Approx. } 14.3 \text { million points) }\end{array}$ & $\begin{array}{c}436(i) \times 325(j) \times 37(k) \\
\text { (Approx. } 5.2 \text { million points) }\end{array}$ \\
\hline $\begin{array}{c}\text { Streamwise }(x) \text { grid spacing }(\Delta x) \\
\text { Spanwise }(y) \text { grid spacing }(\Delta y)\end{array}$ & \multicolumn{2}{|c|}{$(0.035-0.5) \times h$} \\
\hline Vertical $(z)$ grid spacing $(\Delta z)$ & $(0.0001-0.6) \times h$ & $(0.005-1.2) \times h$ \\
\hline
\end{tabular}




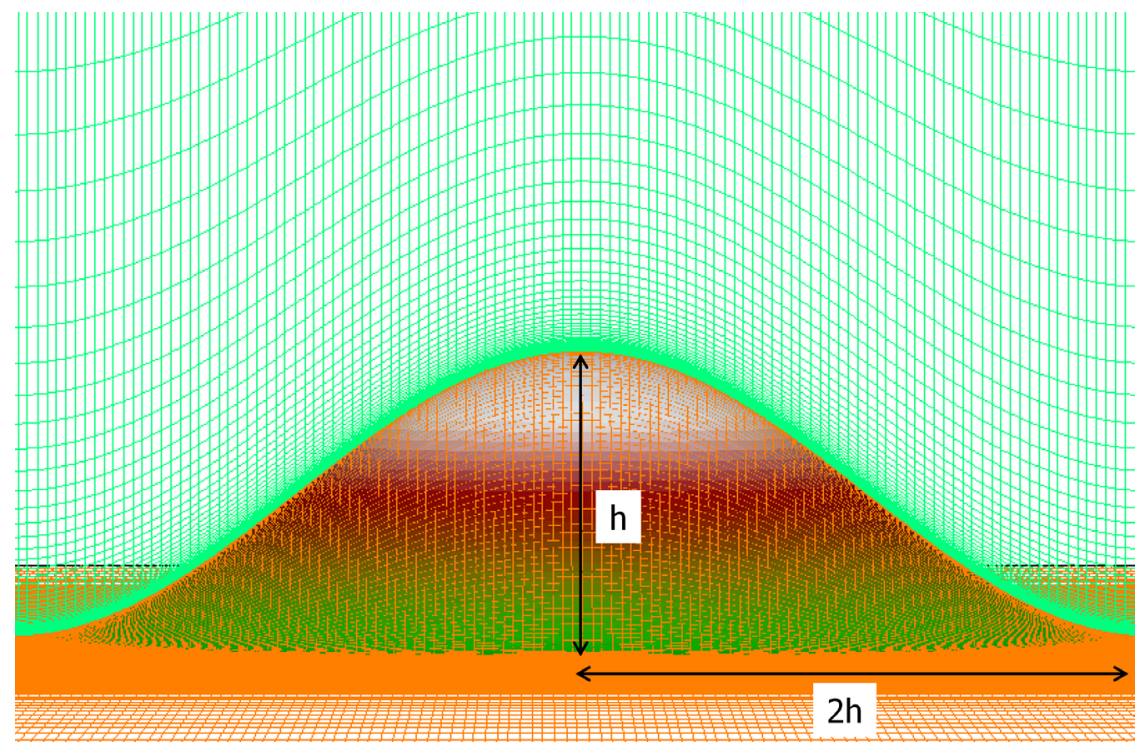

Figure 8. Enlarged view of the computational grid used in the simulations with Meteodyn $\mathrm{WT}, \mathrm{Re}=10^{7}$.

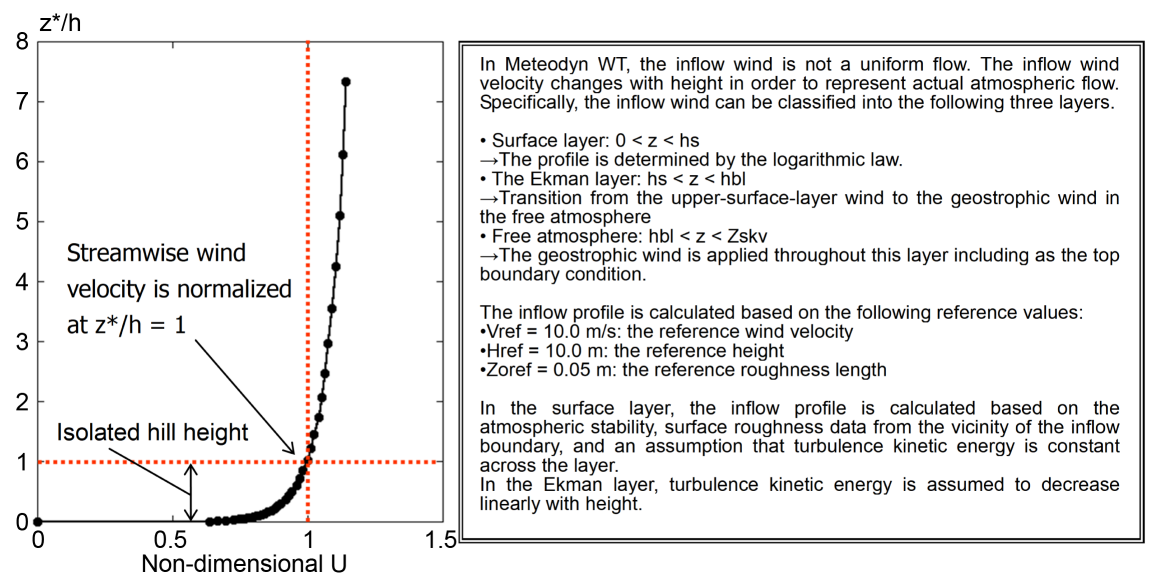

Figure 9. Method adopted in Meteodyn WT for setting the inflow profile and the inflow profile generated for the present study.

Since simulations for a flow with $\operatorname{Re}\left(=U_{\text {in }} h / v\right)=10^{7}$ were not feasible with the RIAM-COMPACT natural terrain version software because of the time step, a numerical wind simulation is performed at $\mathrm{Re}=10^{6}$, which is an order of magnitude smaller than the flow simulated with Meteodyn WT. For this simulation, the number of grid points in the vertical direction is set to 101 (37 for the simulation with Meteodyn WT), and the minimum vertical spacing is set to $\Delta z_{\min } / h=$ $10^{-4}$ based on the Equation (1) $\left(\Delta z_{\min } / h=5.0 \times 10^{-3}\right.$ for the simulation with Meteodyn WT, see Table 2). At the inflow boundary, an inflow profile which is almost identical to the inflow profile used for the simulation with Meteodyn (Figure 9) is used. Free-slip conditions are applied at the side and upper boundaries, and convective outflow conditions are applied at the outflow boundary. At the surfaces of the ground and the isolated hill, non-slip conditions are imposed. The time step is set to $\Delta t=10^{-5} h / U_{\text {in }}$ (refer to Table 2). 


\section{Comparison of the Simulation Results from the Two CFD Software Packages (RIAM-COMPACT and Meteodyn WT) in the Case of a Three-Dimensional, Isolated Hill with a Steep Slope Angle}

Figures 10-12 show results from the simulation with the Meteodyn WT software package (turbulence model: $k$ - $L$ RANS). These results (for a flow at $\mathrm{Re}=$ $10^{7}$ ) indicate that a reverse flow region (vortex region) characterized by negative values of wind velocity does not form downstream of the isolated hill, and a pattern resembling potential flow is present there. Figure 13 shows the results from the simulation with the RIAM-COMPACT natural terrain version software package (turbulence model: the standard Smagorinsky LES). Examinations of the results reveal that a reverse flow region (vortex region) characterized by negative values of wind velocities clearly exists downstream of the isolated hill in the simulated flow at $\operatorname{Re}\left(=U_{\text {in }} h / v\right)=10^{6}$.

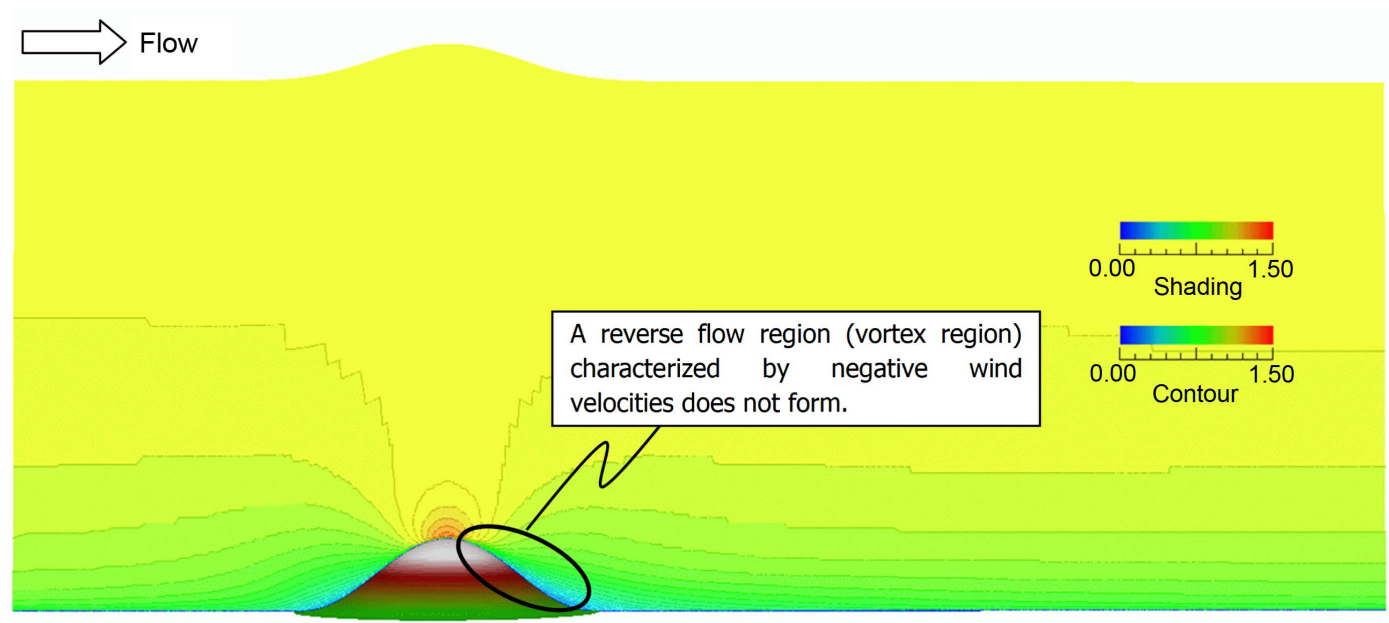

Figure 10. Streamwise $(x)$ wind velocity distribution at the center of the span $(y=0)$, Meteodyn WT, k-L RANS, $\operatorname{Re}=10^{7}$.

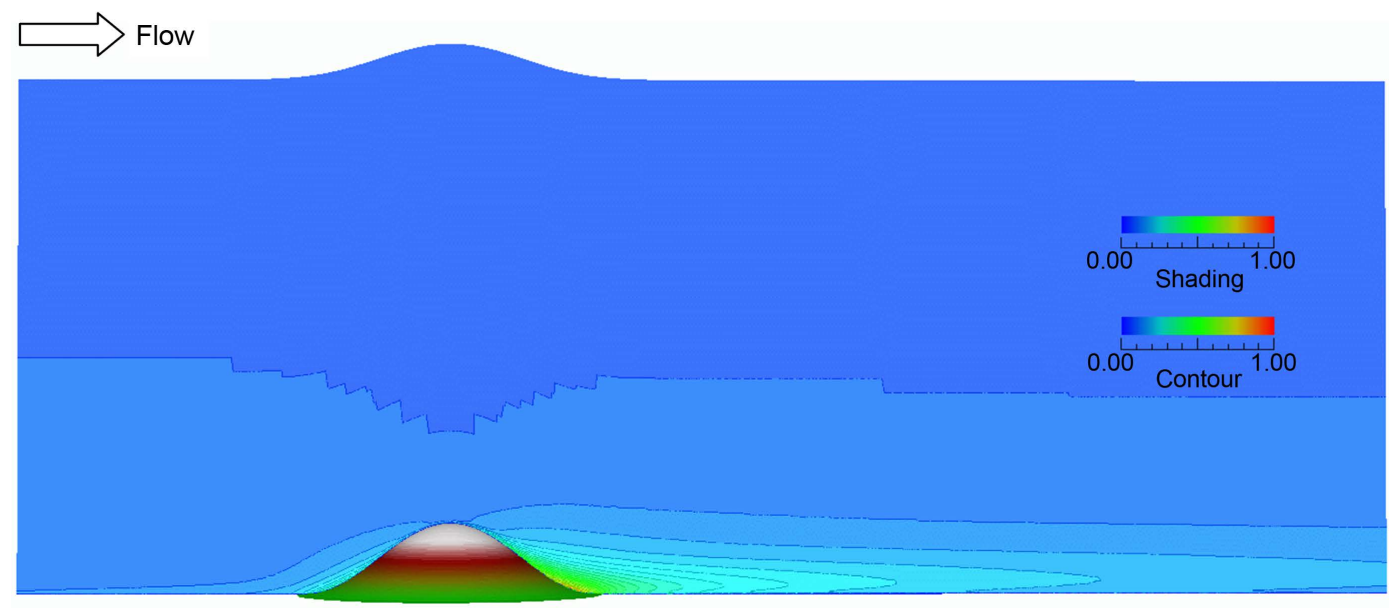

Figure 11. Streamwise (x) turbulence intensity distribution at the center of the span $(y=0)$, Meteodyn WT, $\mathrm{k}-\mathrm{L}$ RANS, $\mathrm{Re}=10^{7}$. 


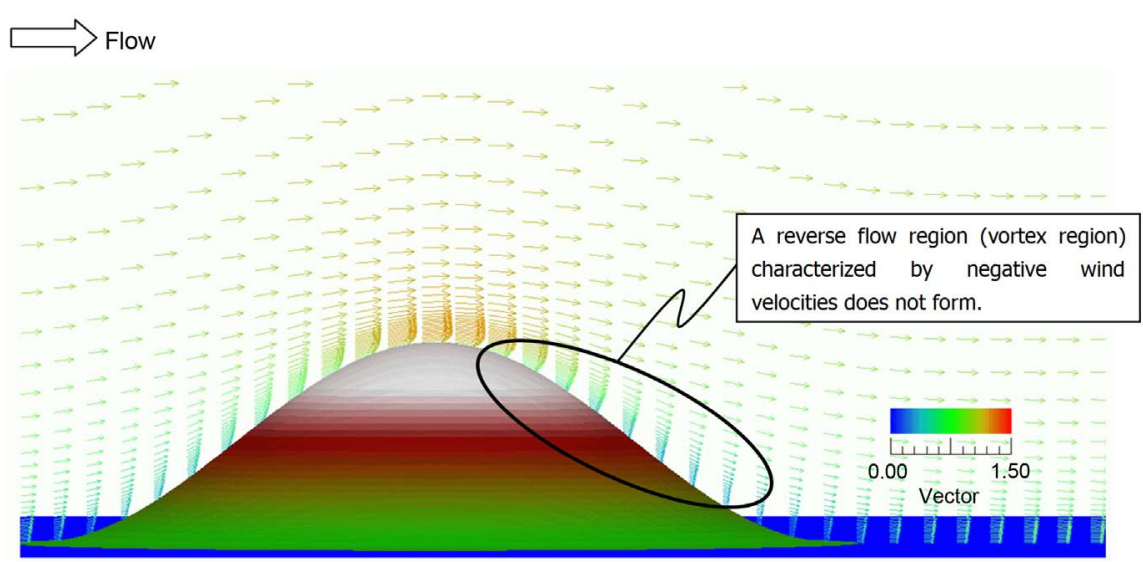

Figure 12. Velocity vectors at the center of the span $(y=0)$ in the vicinity of the isolated hill, Meteodyn WT, k-L RANS model, $\mathrm{Re}=10^{7}$.

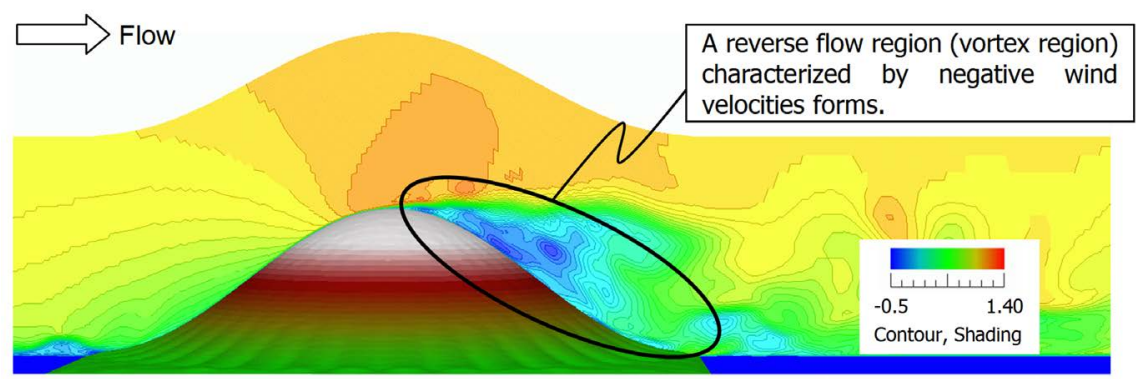

(a)

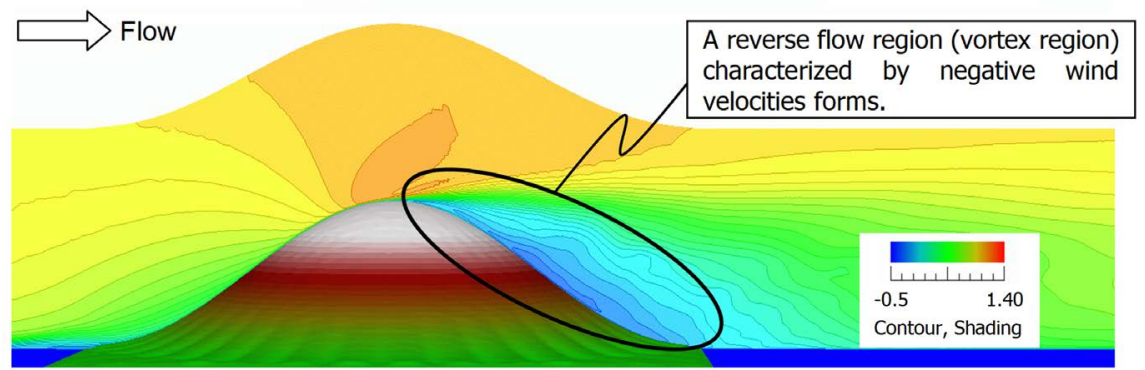

Note: evaluated for $\mathrm{t}=100 \sim 200 \mathrm{~h} / \mathrm{U}_{\text {in }}$

(b)

Figure 13. Streamwise $(x)$ wind velocity distribution at the center of the span $(y=0)$ in the vicinity of the isolated hill, RIAM-COMPACT, standard Smagorinsky LES, Re $=10^{6}$. (a) Instantaneous flow field; (b) Time-averaged flow field.

\section{Comparison of the Simulation Results from the Two CFD Software Packages (RIAM-COMPACT and Meteodyn WT) in the Case of a Large-Scale Wind Farm in China}

Dougu wind farm is located in the city of Mengzi, Honghe prefecture, Yunnan province, China (see Figure 14). The wind farm started operation in 2012 and it consists of 33 Mingyang wind turbines of rated capacity 1.5 MW. The turbines have a hub height of $65 \mathrm{~m}$ with rotor diameter of $82.6 \mathrm{~m}$. The turbines are located on top of a cliff and aligned in a north-south direction with elevation 


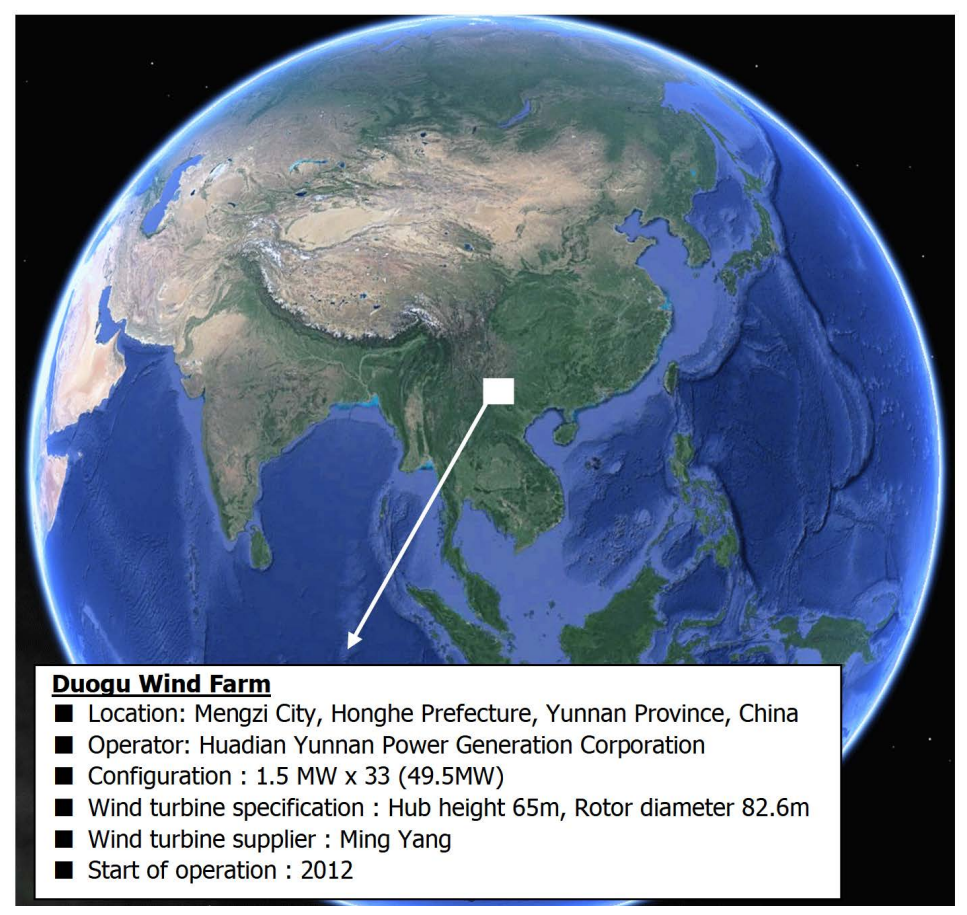

(a)

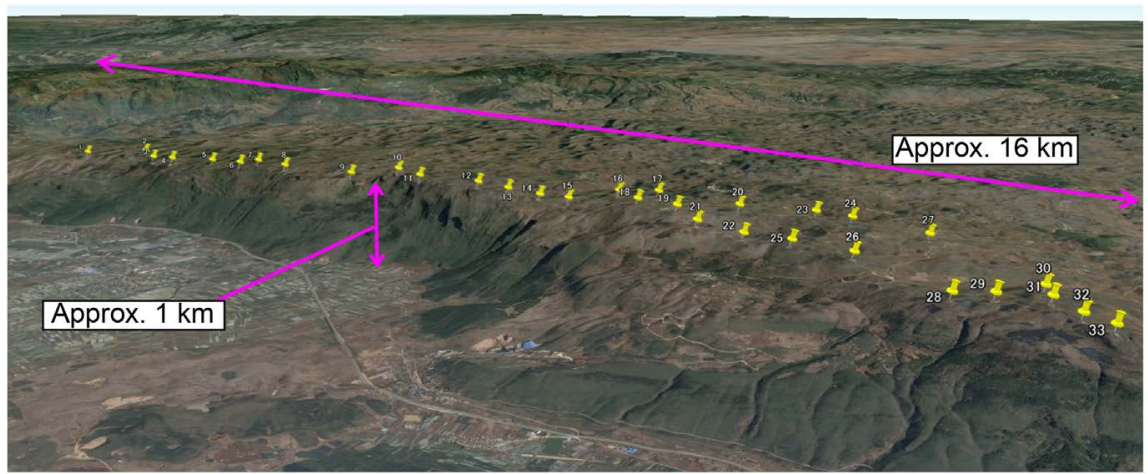

(b)

Figure 14. Large-scale wind farm investigated in the present study (Duogu Wind Farm). (a) Overall view; (b) Enlarged view. A group of 33 wind turbines are located above a steep escarpment.

ranges from around 1850 to 2200 meters. The cliff has a height of around $900 \mathrm{~m}$ with slopes exceeding 60 degrees in places. Aerial photos from Google Earth indicate vegetation is abundant at the bottom of the cliff but scarce along the cliff and in the vicinity of turbines. Since the start of operations, one of the wind turbines, turbine No.12 (T12) has experienced vibration problems. Wind farm operator Yunnan Huadian Dougu Wind Power Corporation (YUDWPC) suspected the vibration issue is related to wind conditions. In the present study, the simulations are performed with RIAM-COMPACT, which is based on an LES turbulence model, and WindSim, which is based on a RANS turbulence model. The results from the simulations are compared.

The vibration problem of turbine T12 was investigated by the operator 
YUDWPC and a report was issued in April 2014 [28]. Stated in the report was that high vibration data was recorded only when the wind was blowing from the southwest. Wind direction on the ground level was observed to be in the reverse direction from that recorded by the nacelle anemometer. Analysis of the vibration data indicates the vibration is in the vertical direction. This suggests the vibration is associated with abnormal vertical wind shear across the wind turbine rotor. As shown in Figure 15, a figure extracted from the report, it was deduced that the presence of the small hill located about $150 \mathrm{~m}$ upstream from turbine T12 was causing the onset of turbulence and reverse flow which led to the vibration recorded.

For LES simulation, the RIAM-COMPACT natural terrain version software package was employed. The software uses a standard Smagorinsky turbulence model. For the simulation, SRTM $90 \mathrm{~m}$ data was used for elevation data. Wind direction is set to true north at 247 degrees and the computational domain constructed is shown in Figure 16 with the following details:

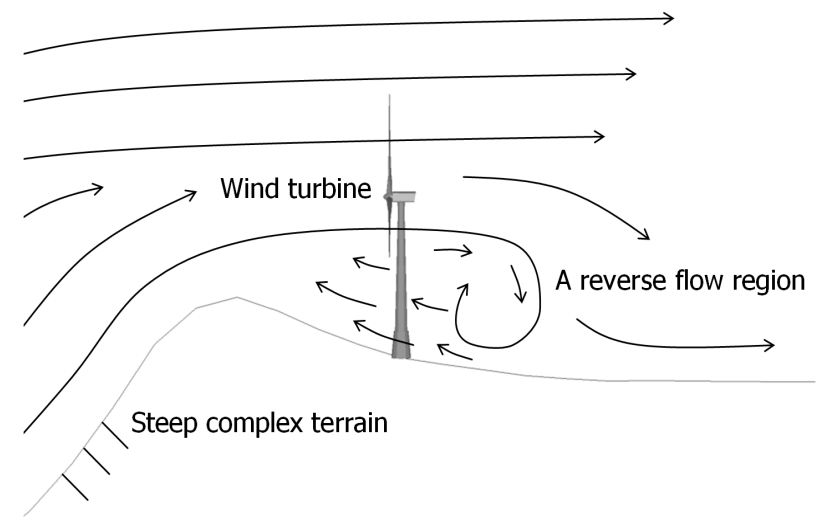

Figure 15. Deduction made on the airflow upstream and in the vicinity of turbine T12.

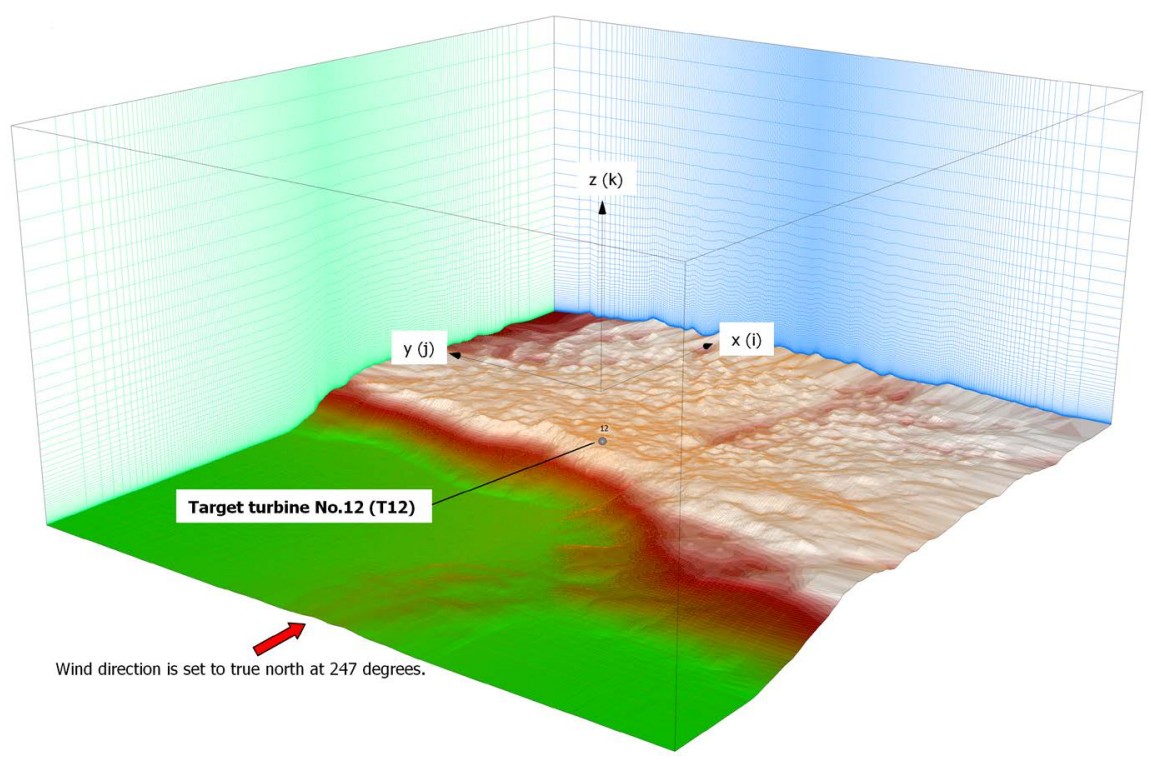

Figure 16. Computational domain and grid used for the simulation with RIAM-COMPACT. 
- Domain size: $14.0 \mathrm{~km} \times 13.3 \mathrm{~km} \times 8.3 \mathrm{~km}$

- Elevation: $1275 \mathrm{~m}$ (Min) - $2232 \mathrm{~m}$ (Max)

- Calculation grid points: $300 \times 400 \times 60$

- Total number of grid points: 7.2 million

- Grid spacing: $8 \mathrm{~m}$ - $957 \mathrm{~m}(x), 13 \mathrm{~m}$ - $72 \mathrm{~m}(y), 1 \mathrm{~m}-470 \mathrm{~m}(z)$

To increase calculation accuracy, the mesh is concentrated around the turbine positions in both the $x$ and $y$ direction as shown in Figure 16. No roughness consideration is given in the present LES simulation. Atmospheric stability is set to neutral stability. After the calculation has been stabilized, numerical results in the calculation domain are output for a real time of ten minutes with an interval of one second.

Figure 17 shows an instantaneous vector plot across turbine T12. This picture clearly shows flow separation occurred at the small hill located $140 \mathrm{~m}$ upstream from the turbine, and the onset of the formation of the recirculating vortex behind the hill. The turbulent flow extends downstream forming a reverse flow region characterized by negative values of wind speed covering the lower part of the wind turbine rotor.

The simulation results also indicate that the wind flow is relatively undisturbed above hub height level. The $U$ component wind speed time series during the ten minute simulation at rotor top $(106.3 \mathrm{~m})$, hub center $(65 \mathrm{~m})$, rotor bottom $(23.7 \mathrm{~m})$ and surface level $(10 \mathrm{~m})$ positions are plotted in Figure 18.

Referring to Figure 18 , it is obvious that the wind speed at surface $(10 \mathrm{~m})$ and rotor bottom is significantly lower and showing more fluctuations than the wind speed at the hub and top part of the rotor. Wind speed varies between 15.0 to $20.0 \mathrm{~m} / \mathrm{s}$ at hub height and rotor top whereas for rotor bottom wind speed fluctuates between negative $6.2 \mathrm{~m} / \mathrm{s}$ to $2.0 \mathrm{~m} / \mathrm{s}$. Negative wind speed indicates the

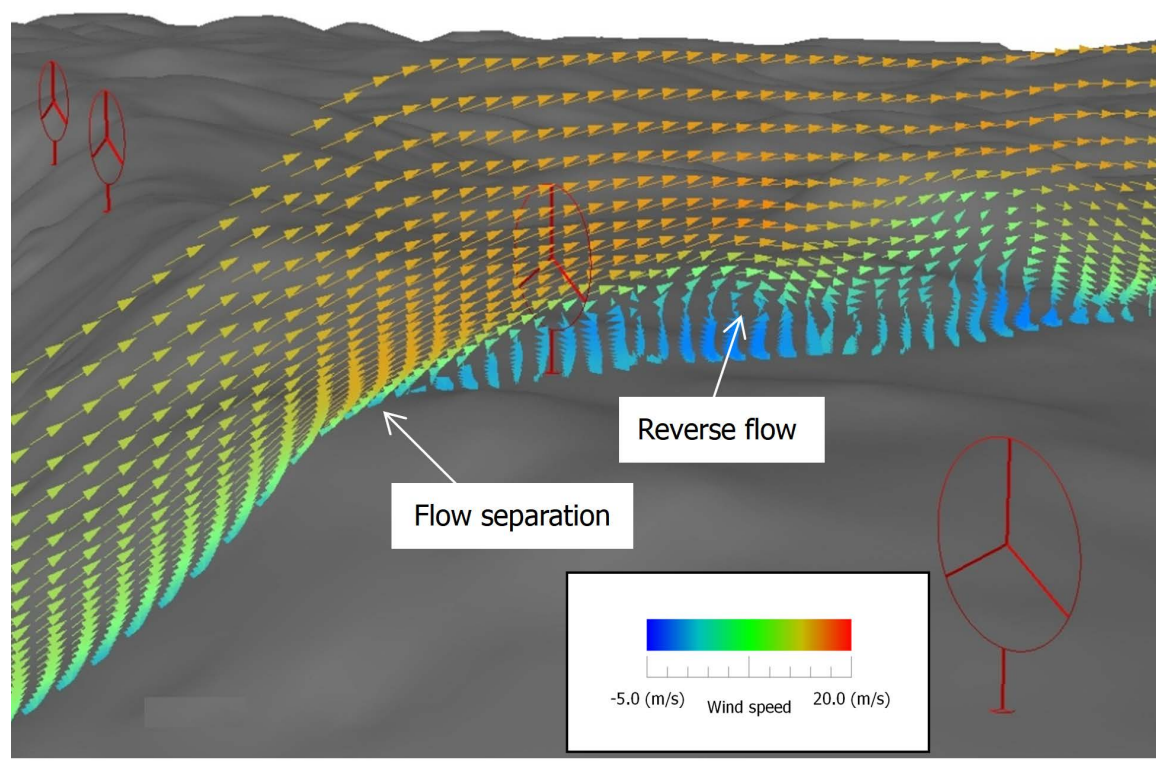

Figure 17. Instantaneous vector plot at turbine T12. 


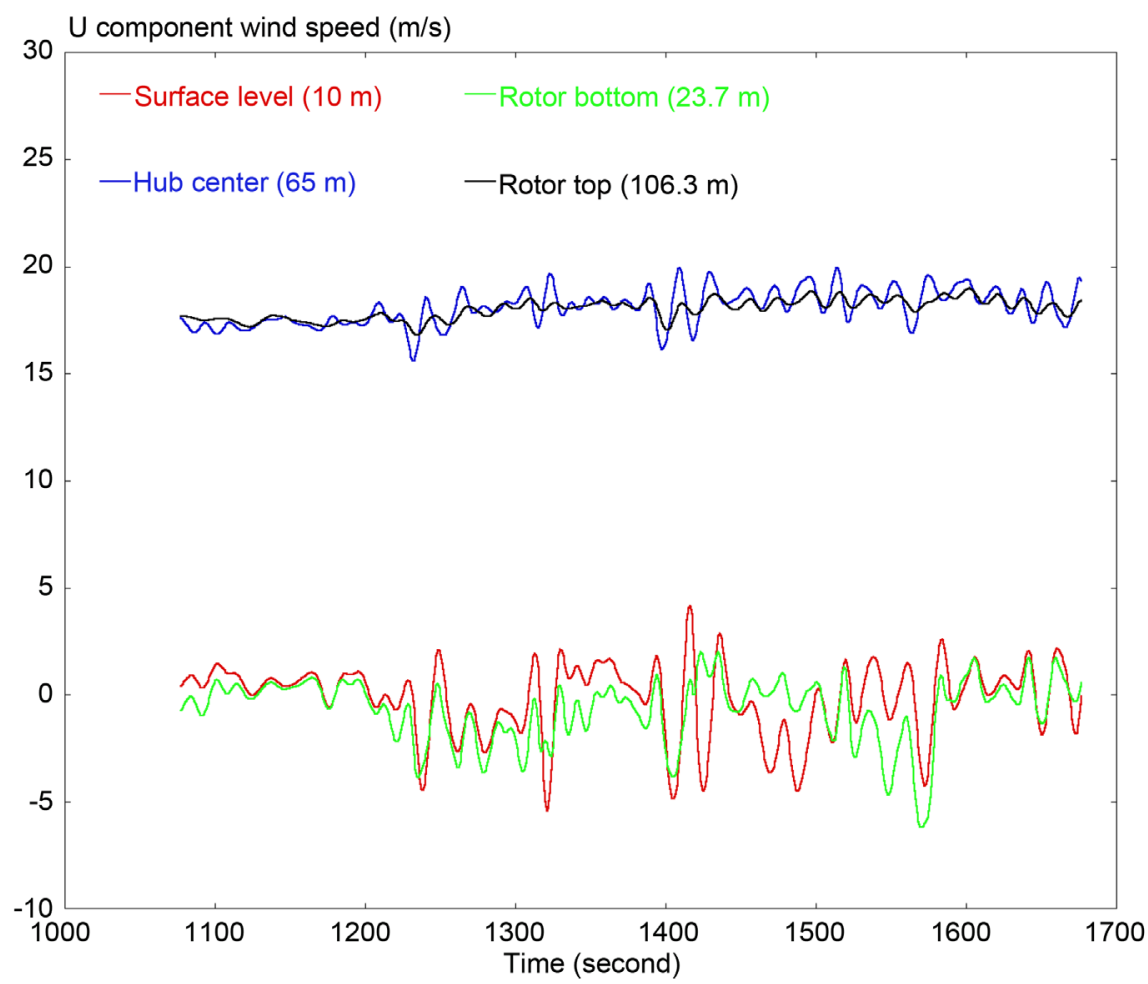

Figure 18. Time series of $U$ component wind speed at rotor top, hub, rotor bottom and ground surface level at turbine T12.

wind is flowing in reverse direction. During the ten minute simulation, negative values account for $62 \%$ of the total data at rotor bottom. When the rotor bottom wind speed is at its minimum of negative $6.2 \mathrm{~m} / \mathrm{s}$ the wind speed at rotor top is at $18.1 \mathrm{~m} / \mathrm{s}$, hence a very large absolute wind speed difference of $24.3 \mathrm{~m} / \mathrm{s}$. Excluding the negative wind speed data, the wind speed difference across the rotor face (between rotor top and rotor bottom) has a maximum value of $18.7 \mathrm{~m} / \mathrm{s}$ and an average of $17.4 \mathrm{~m} / \mathrm{s}$. The maximum wind shear exponent is calculated to be 5.8 with an average value of 2.5, far exceeding the IEC standard average shear value of 0.2 .

The average, minimum and maximum values of the $U$ component wind speed at turbine T12 are shown in Figure 19. The average values represent the average shear profile seen at turbine T12. Referring to that, the reverse flow region results in a negative or very low wind speed from ground surface level to the rotor bottom of around $25 \mathrm{~m}$. Wind speed increases gradually from $25 \mathrm{~m}$ and starts leveling off at around $50 \mathrm{~m}$.

In this study, the commercial software Meteodyn WT (turbulence model: $k-L$ RANS) was employed and its results were compared with the results calculated by the RIAM-COMAPCT. The calculation parameters are shown in Table 3. The calculation domain is a radius of $10 \mathrm{~km}$ for the $x-y$ direction with turbine T12 as center; $z$ direction has a maximum of $200 \mathrm{~m}$. Wind Direction is set at 247 degrees with minimum vertical and horizontal resolution set to $5 \mathrm{~m}$ and $2 \mathrm{~m}$ respectively. Atmospheric stability is set to neutral. The calculation was completed smoothly with computation convergence recoded at $99.3 \%$. 


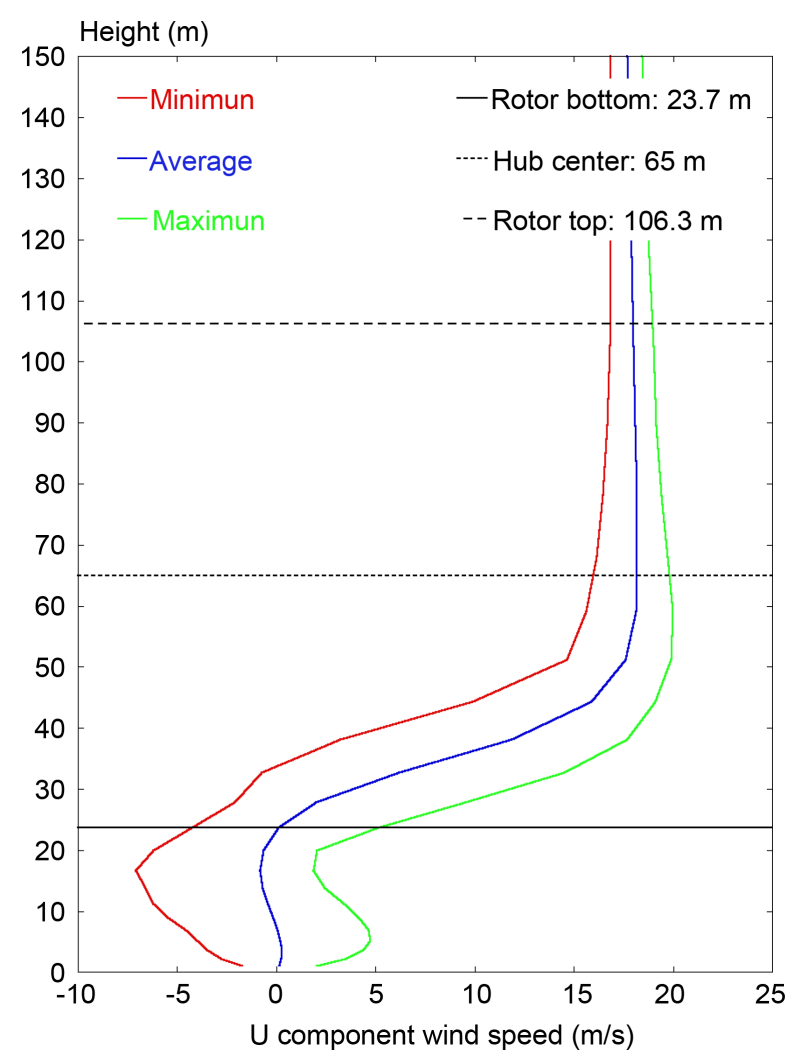

Figure 19. Vertical shear profile predicted by RIAM-COMAPCT, average, minimum and maximum of $U$ component wind speed variation with height.

Table 3. Numerical simulation methods, parameters, and settings for Meteodyn WT.

\begin{tabular}{cc} 
Wind direction & 247 degrees \\
Thermal stability class & 2 \\
Smoothing-Whole domain & 1 \\
Forest model & Robust model \\
Minimum horizontal spacing & $5 \mathrm{~m}$ \\
Minimum vertical spacing & $2 \mathrm{~m}$ \\
Horizontal expansion coefficient & 1.1 \\
Vertical expansion coefficient & 1.2 \\
Grid points & $225(\mathrm{i}) \times 237(\mathrm{j}) \times 44(\mathrm{k})$ \\
Maximum iteration number & 25 \\
Convergence & $99.3 \%$ \\
\hline
\end{tabular}

Meteodyn WT's calculation output includes the speed-up factor from height $20 \mathrm{~m}$ to $200 \mathrm{~m}$ at an interval of $20 \mathrm{~m}$ at turbine T12. These values are shown in Table 4. The speed-up factor is the wind speed ratio at the given height referencing the wind speed at height $10 \mathrm{~m}$. The speed-up factor therefore resembles the vertical shear profile. Assuming a wind speed of $9.5 \mathrm{~m} / \mathrm{s}$ at $10 \mathrm{~m}$ height, wind 
Table 4. Speed-up factor at turbine T12 and calculated wind speed.

\begin{tabular}{ccc}
\hline Height $(\mathrm{m})$ & Speed-up factor & Wind speed $(\mathrm{m} / \mathrm{s})$ \\
\hline 20 & 1.850 & 17.58 \\
40 & 1.952 & 18.54 \\
60 & 2.002 & 19.02 \\
80 & 2.013 & 19.12 \\
100 & 2.008 & 19.08 \\
120 & 1.998 & 18.98 \\
140 & 1.987 & 18.88 \\
160 & 1.977 & 18.78 \\
180 & 1.968 & 18.70 \\
200 & 1.959 & 18.61 \\
\hline
\end{tabular}

speed at different heights can be calculated based on the speed-up factor and the results are shown in Table 4.

The wind speed figures in Table 4 are plotted in Figure 20 and the resulting wind shear profile is compared with the shear profile (average values) predicted by RIAM-COMPACT. It can be seen from Figure 20 that the shapes of the two profiles are similar from $50 \mathrm{~m}$ upwards but distinctively different below $50 \mathrm{~m}$. Meteodyn WT does not seem to predict any flow separation and reverse flow region and therefore there is no significant wind speed reduction between $25 \mathrm{~m}$ and $50 \mathrm{~m}$, and also no negative wind speed values below $25 \mathrm{~m}$ as predicted by RIAM-COMPACT. Numerical comparison results are shown in Table 5.

Referring to Table 5, across the wind turbine rotor face, RIAM-COMPACT predicted a large wind speed difference with a shear exponent exceeding the IEC standard value of 0.2 by a large margin. In sharp contrast, Meteodyn WT predicted a small wind speed difference with a shear exponent of 0.025 which is significantly below the IEC standard.

\section{Summary}

Simulations were performed for airflow around a three-dimensional, isolated hill with a steep slope angle in order to compare the flow pattern simulated in the vicinity of the hill by three software packages. For the simulations, three software packages were used: 1) WindSim (turbulence model: RNG $k-\mathcal{E}$ RANS), 2) Meteodyn WT (turbulence model: $k-L$ RANS), which are the leading commercially available CFD software packages in the wind power industry and 3) RIAM-COMPACT (turbulence model: standard Smagorinsky LES). Comparisons of the simulated results revealed a distinct difference in the simulated flow patterns in the vicinity of the isolated hill (especially downstream of the hill). No reverse flow region (vortex region) characterized by negative wind velocities was identified downstream of the isolated hill in the result from the simulation with WindSim (RNG $k-\mathcal{E}$ RANS) and Meteodyn WT ( $k-L$ RANS). In the case 


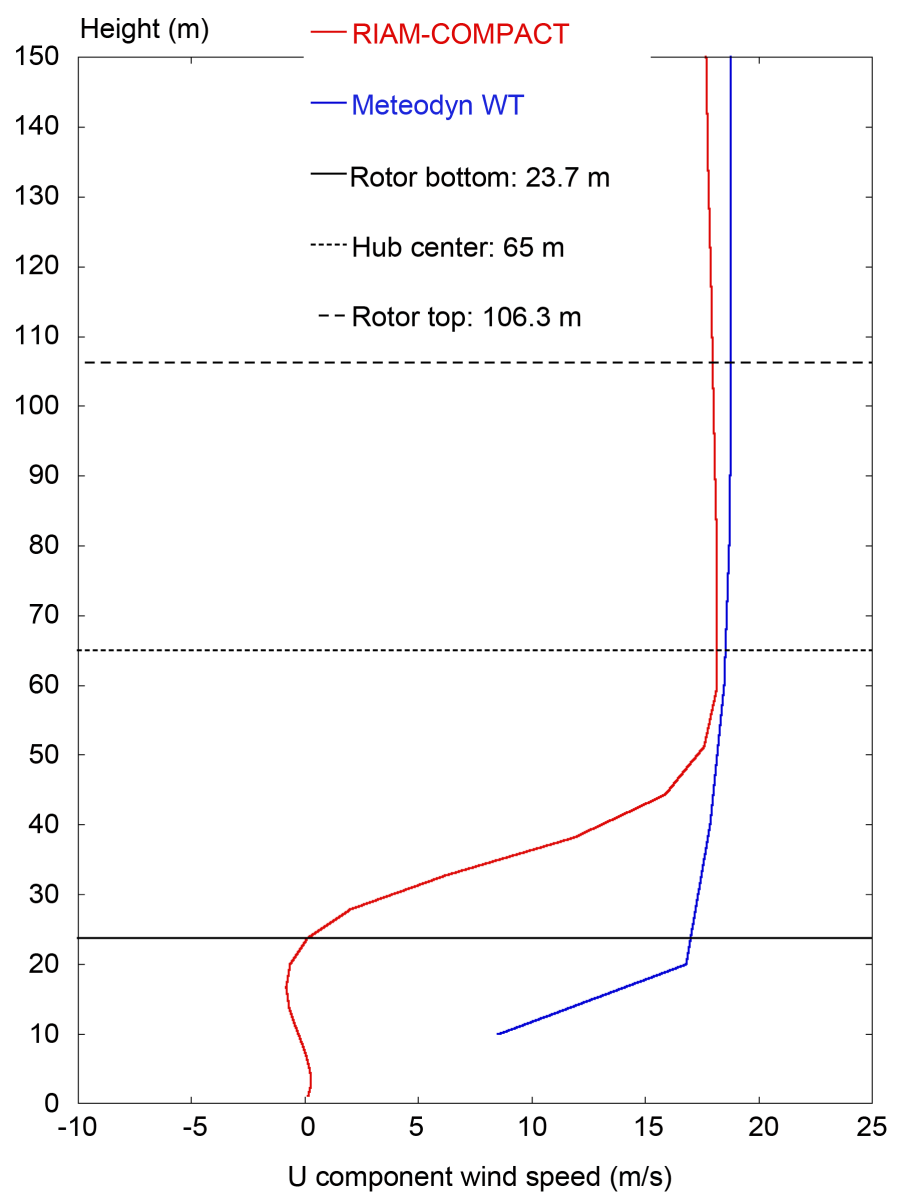

Figure 20. Comparison of vertical shear profile between the wind flows simulated by RIAM-COMPACT and Meteodyn WT.

Table 5. Numerical comparison of vertical shear profile between RIAM-COMPACT and Meteodyn WT.

\begin{tabular}{ccc}
\hline & RIAM-COMPACT & Meteodyn WT \\
Wind speed near rotor bottom at height $(\mathrm{m} / \mathrm{s})$ & $0.11(23.7 \mathrm{~m})$ & $17.58(20 \mathrm{~m})$ \\
Wind speed near rotor top at height $(\mathrm{m} / \mathrm{s})$ & $18.0(102.6 \mathrm{~m})$ & $19.08(100 \mathrm{~m})$ \\
Wind speed difference $(\mathrm{m} / \mathrm{s})$ & 17.9 & 1.5 \\
Average shear exponent & 3.5 & 0.025 \\
Average shear exponent exceeding IEC standard & YES & NO
\end{tabular}

of the simulation with RIAM-COMPACT (standard Smagorinsky LES), a reverse flow region (vortex region) characterized by negative wind velocities clearly forms.

Next, a turbine which has vibration problems was simulated using the Meteodyn WT ( $k$ - $L$ RANS) and RIAM-COMPACT (standard Smagorinsky LES). It was deduced from the vibration and turbine operation data that a possible reverse flow region near the rotor bottom was the direct cause of the vibration. Simulation results from LES-based code RIAM-COMPACT predict a flow sepa- 
ration upstream and a reverse flow region at the rotor bottom. Simulation results from RANS-based software Meteodyn WT produced a very different shear profile which suggests the reverse flow and the associated flow separation were not predicted.

\section{Acknowledgements}

This work was supported by JSPS KAKENHI Grant Number 17H02053.

\section{Conflicts of Interest}

The authors declare no conflicts of interest regarding the publication of this paper.

\section{References}

[1] Uchida, T. (2018) Design Wind Speed Evaluation Technique in Wind Turbine Installation Point by Using the Meteorological and CFD Models. Journal of Flow Control, Measurement \& Visualization, 6, 168-184. https://doi.org/10.4236/jfcmv.2018.63014

[2] Uchida, T. (2018) Computational Investigation of the Causes of Wind Turbine Blade Damage at Japan's Wind Farm in Complex Terrain. Journal of Flow Control, Measurement \& Visualization, 6, 152-167. https://doi.org/10.4236/jfcmv.2018.63013

[3] Uchida, T. (2018) Computational Fluid Dynamics Approach to Predict the Actual Wind Speed over Complex Terrain. Energies, 11, 1694. https://doi.org/10.3390/en11071694

[4] Uchida, T. (2018) LES Investigation of Terrain-Induced Turbulence in Complex Terrain and Economic Effects of Wind Turbine Control. Energies, 11, 1530. https://doi.org/10.3390/en11061530

[5] Uchida, T. (2018) Computational Fluid Dynamics (CFD) Investigation of Wind Turbine Nacelle Separation Accident over Complex Terrain in Japan. Energies, 11, 1485. https://doi.org/10.3390/en11061485

[6] Uchida, T. (2018) Large-Eddy Simulation and Wind Tunnel Experiment of Airflow over Bolund Hill. Open Journal of Fluid Dynamics, 8, 30-43.

https://doi.org/10.4236/ojfd.2018.81003

[7] Uchida, T. (2017) High-Resolution LES of Terrain-Induced Turbulence around Wind Turbine Generators by Using Turbulent Inflow Boundary Conditions. Open Journal of Fluid Dynamics, 7, 511-524. https://doi.org/10.4236/ojfd.2017.74035

[8] Uchida, T. (2017) High-Resolution Micro-Siting Technique for Large Scale Wind Farm Outside of Japan Using LES Turbulence Model. Energy and Power Engineering, 9, 802-813. https://doi.org/10.4236/epe.2017.912050

[9] Uchida, T. and Ohya, Y. (2011) Latest Developments in Numerical Wind Synopsis Prediction Using the RIAM-COMPACT CFD Model-Design Wind Speed Evaluation and Wind Risk (Terrain-Induced Turbulence) Diagnostics in Japan. Energies, 4, 458-474. https://doi.org/10.3390/en4030458

[10] Uchida, T., Ohya, Y. and Sugitani, K. (2011) Comparisons between the Wake of a Wind Turbine Generator Operated at Optimal Tip Speed Ratio and the Wake of a Stationary Disk. Modelling and Simulation in Engineering, 2011, Article ID: 749421. https://doi.org/10.1155/2011/749421 
[11] Uchida, T., Maruyama, T. and Ohya, Y. (2011) New Evaluation Technique for WTG Design Wind Speed Using a CFD-Model-Based Unsteady Flow Simulation with Wind Direction Changes. Modelling and Simulation in Engineering, 2011, Article ID: 941870. https://doi.org/10.1155/2011/941870

[12] Uchida, T. and Ohya, Y. (2008) Micro-Siting Technique for Wind Turbine Generators by Using Large-Eddy Simulation. Journal of Wind Engineering \& Industrial Aerodynamics, 96, 2121-2138. https://doi.org/10.1016/j.jweia.2008.02.047

[13] Uchida, T. and Ohya, Y. (2001) Large-Eddy Simulation of Turbulent Airflow over Complex Terrain. Journal of Wind Engineering and Industrial Aerodynamics, 91, 219-229. https://doi.org/10.1016/S0167-6105(02)00347-1

[14] https://mdx.plm.automation.siemens.com/star-ccm-plus

[15] http://www.ansys.com/

[16] Uchida, T. (2016) Reproducibility of Complex Turbulent Flow Using Commercially-Available CFD Software-Report 3: For the Case of a Three-Dimensional Cube. Reports of Research Institute for Applied Mechanics, Kyushu University, Fukuoka, No. 150, 71-83. https://doi.org/10.15017/1660835

[17] Uchida, T. (2016) Reproducibility of Complex Turbulent Flow Using Commercially-Available CFD Software-Report 2: For the Case of a Two-Dimensional Ridge with Steep Slopes. Reports of Research Institute for Applied Mechanics, Kyushu University, Fukuoka, No. 150, 60-70. https://doi.org/10.15017/1660834

[18] Uchida, T. (2016) Reproducibility of Complex Turbulent Flow Using Commercially-Available CFD Software-Report 1: For the Case of a Three-Dimensional Isolated-Hill with Steep Slopes. Reports of Research Institute for Applied Mechanics, Kyushu University, Fukuoka, No. 150, 47-59. https://doi.org/10.15017/1660833

[19] http://www.openfoam.com/

[20] http://www.gnu.org/licenses/gpl-3.0.en.html

[21] Uchida, T. (2017) CFD Prediction of the Airflow at a Large-Scale Wind Farm above a Steep, Three-Dimensional Escarpment. Energy and Power Engineering, 9, 829-842. https://doi.org/10.4236/epe.2017.913052

[22] https://meteodyn.com/en/

[23] https://www.windsim.com/

[24] Kim, J. and Moin, P. (1985) Application of a Fractional-Step Method to Incompressible Navier-Stokes Equations. Journal of Computational Physics, 59, 308-323. https://doi.org/10.1016/0021-9991(85)90148-2

[25] Kajishima, T. (1994) Upstream-Shifted Interpolation Method for Numerical Simulation of Incompressible Flows. Bulletin of JSME, 60, 3319-3326. (In Japanese) https://doi.org/10.1299/kikaib.60.3319

[26] Kawamura, T., Takami, H. and Kuwahara, K. (1986) Computation of High Reynolds Number Flow around a Circular Cylinder with Surface Roughness. Fluid Dynamics Research, 1, 145-162. https://doi.org/10.1016/0169-5983(86)90014-6

[27] Smagorinsky, J. (1963) General Circulation Experiments with the Primitive Equations, Part 1, Basic Experiments. Monthly Weather Review, 91, 99-164. https://doi.org/10.1175/1520-0493(1963)091<0099:GCEWTP>2.3.CO;2

[28] Yunnan Huadian Dougu Wind Power Corporation (2014) Temporary Measures for Safe Operation for Wind Turbine No. 7 and No. 12 of Dougu Wind Farm. 


\section{Appendix}

As discussed in the above text of the present paper, no reverse flow region (vortex region) characterized by negative wind velocities was identified downstream of the isolated hill in the result from the simulation with WindSim (RNG $k$ - $\mathcal{E}$ RANS) and Meteodyn WT ( $k$ - $L$ RANS), in which the Reynolds number was set to $\operatorname{Re}=10^{7}$ (the default value for Meteodyn WT). Accordingly, in order to investigate if a flow pattern similar to that simulated with Meteodyn WT ( $k-L$ RANS) can also be formed with the use of RIAM-COMPACT (standard Smagorinsky LES), a simulation is conducted with RIAM-COMPACT in which the method for setting the surface boundary conditions is modified. Specifically, the values of the three components of the wind velocity from $k=2$ on the computational grid of Meteodyn WT ( $k$-L RANS) ( $k=1$ corresponds to the surface of the ground or the isolated hill in Meteodyn WT) are assigned as the values for the surface boundary conditions $(k=1)$ for the simulation with RIAM-COMPACT (standard Smagorinsky LES) (Figure 21). That is, this simulation is one in which non-zero values are assigned for the wind velocity at the surfaces of the ground and the isolated hill at all computational steps (Dirichlet boundary condition). The Reynolds number, based on the height of the isolated hill, is set to Re $\left(=U_{\text {in }} h / v\right)=10^{4}$, and the computational grids shown in Figure 7 and Figure 8 are used. For the inflow profile, a uniform flow profile is adopted in which the wind velocity does not change in the vertical direction. The other boundary conditions are set using the same methods as those discussed in the main text of the present paper. The time step is set to $\Delta t=2 \times 10^{-3} \mathrm{~h} / U_{\text {in. }}$. The results obtained from this simulation are compared to those from a simulation which is identical to the simulation described in this addendum, except that non-slip conditions are applied at the surfaces of the ground and the isolated hill, that is, the three components of the wind velocity are all set to zero as Dirichlet boundary conditions. For convenience, the simulation in which non-zero wind velocities are applied as a Dirichlet boundary condition is denoted as Case 1, and the simulation in which all three components of the wind velocity are set to zero is denoted as Case 2. A comparison of the simulation results is shown in Figure 22.

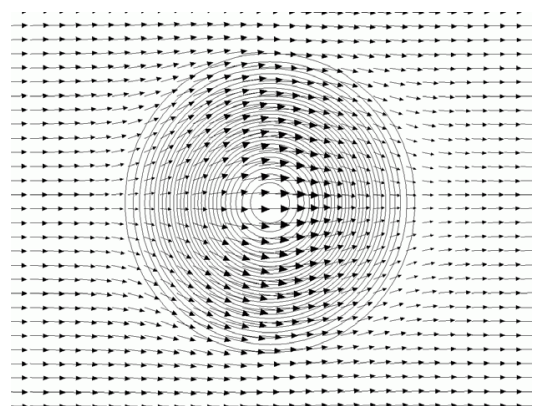

Figure 21. Velocity vectors at the surfaces of the ground and the isolated hill $(\mathrm{k}=1)$, simulation for which the wind velocity from $\mathrm{k}=2$ in Meteodyn WT is used as a Dirichlet boundary condition, Case 1 . 

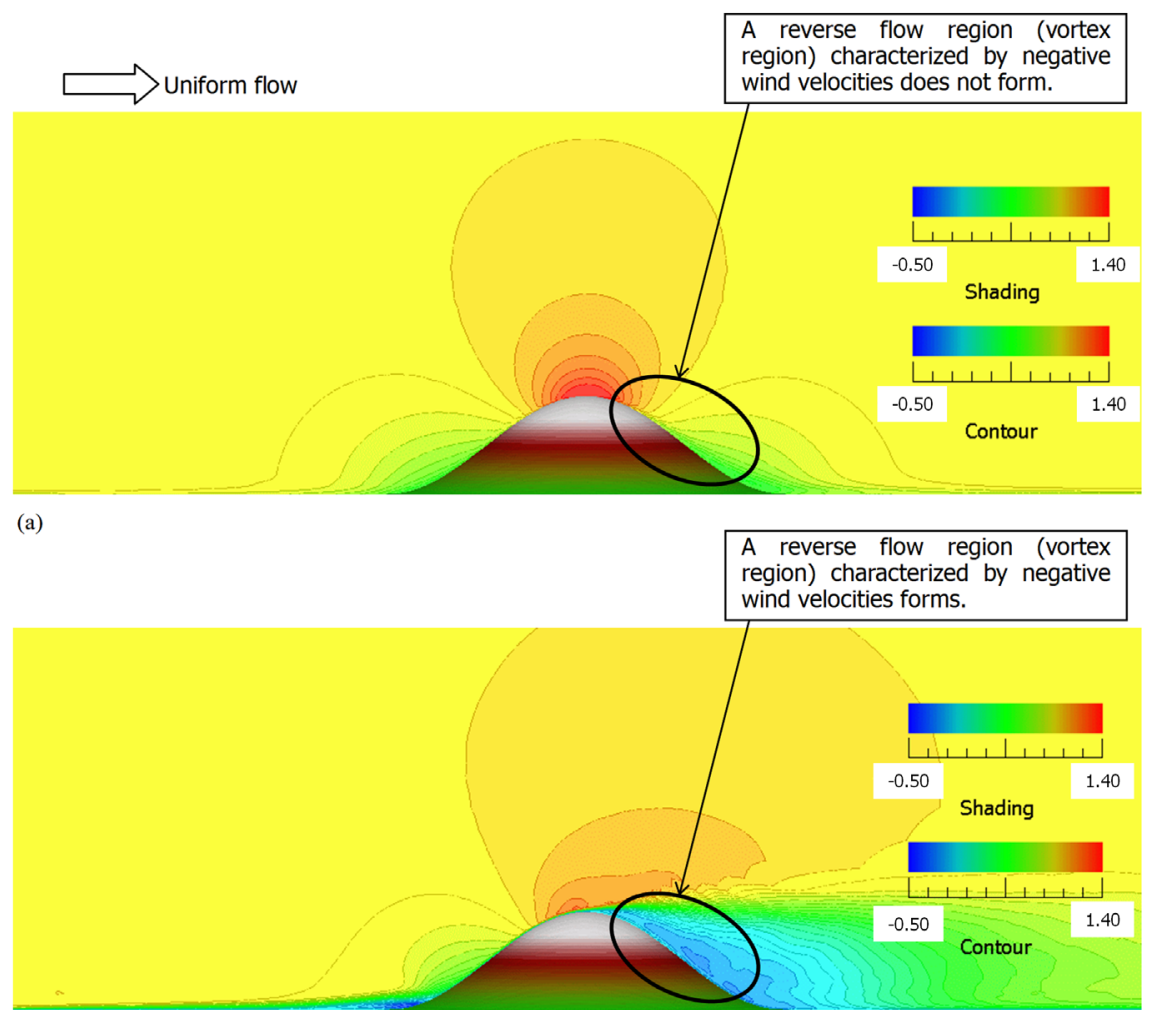

(b)

Figure 22. Streamwise (x) wind velocity distribution in the vicinity of the isolated hill at the center of the span $(y=0)$, RIAM-COMPACT, standard Smagorinsky LES, $\operatorname{Re}=10^{4}$. (a) Case 1: Simulation result from the case in which non-zero wind velocities are appliedas a Dirichlet boundary condition; (b) Case 2: Simulation result from the case in which all three wind velocity components are set to zero as a Dirichlet boundary condition.

An examination of Figure 22 reveals that no reverse flow region (vortex region) characterized by negative wind velocities forms downstream of the isolated hill in Case 1, in which non-zero wind velocities are applied as a Dirichlet boundary condition. In this case, a flow pattern resembling potential flow exists downstream of the isolated hill instead. In contrast, in Case 2, in which all three components of the wind velocity are set to zero as a Dirichlet boundary condition, a distinct reverse flow region (vortex region) forms downstream of the isolated hill.

Thus, the flow pattern which forms in the vicinity of the isolated hill varies significantly according to the velocity boundary conditions applied for the surfaces of the ground and the isolated hill. 